\title{
Backpressure-based Routing Protocol for DTNs
}

\author{
Amit Dvir \\ Department of Computer Science \\ The College of Management Academic Studies \\ Israel \\ azdvir@gmail.com
}

\author{
Athanasios V. Vasilakos \\ Department of Computer and \\ Telecommunications Engineering \\ University of Western Macedonia \\ Greece \\ vasilako@ath.forthnet.gr
}

\begin{abstract}
In this paper we consider an alternative, highly agile approach called backpressure routing for Delay Tolerant Networks (DTN), in which routing and forwarding decisions are made on a per-packet basis. Using information about queue backlogs, random walk and data packet scheduling nodes can make packet routing and forwarding decisions without the notion of end-to-end routes. To the best of our knowledge, this is the first ever implementation of dynamic backpressure routing in DTNs. Simulation results show that the proposed approach has advantages in terms of DTN networks.
\end{abstract}

\section{Categories and Subject Descriptors}

C.2.2 [Network Protocols]: Routing protocols

\section{General Terms}

Algorithms

\section{Keywords}

Delay Tolerant Network, Backpressure approach

\section{INTRODUCTION}

Traditionally, communication networks (wired or wireless) have always been assumed to be connected almost all the time, i.e., at least one end-to-end path between any pair of nodes in the network exists most of the time. However, for some emerging applications such as emergency response, habitat monitoring, and VANETs, the assumption of Şuniversal connectivity $\breve{T}$ no longer holds. Networked environments that operate under such intermittent connectivity are also referred to as episodically connected, delay tolerant, or disruption tolerant networks (DTNs). Clearly, traditional routing cannot deliver adequate performance in DTNs. In civilian DTNs, where the nodes are human/car/train, a given node may visit some locations (e.g., a personŠs home or office), which often exemplify spatial correlation of movement. Also, a given node may exhibit different mobility behaviors depending on the time of day (temporal correlation). Moreover, most people are selfish. As a result, in civilian DTNs such as the Pocket Switched Network, a node may not be willing to forward packets for others.

Copyright is held by the author/owner(s).

SIGCOMM'10, August 30-September 3, 2010, New Delhi, India. ACM 978-1-4503-0201-2/10/08.
Random walks are a natural approach to graph exploration. In its simplest form, a packet is (at each step) forwarded to a node chosen randomly and uniformly from the current nodeŠs neighbors. Moreover, random walks have also been used to achieve load balancing in multi-path routing environments. We explore in this work an exciting alternative approach: dynamic backpressure routing [2] for DTNs. Backpressure routing allows for greater responsiveness to link variation, queue hot-spots, and node mobility; this substantially enhances the throughput efficiency of collection. Backpressure routing refers to techniques grounded in stochastic network optimization, referred to as Utility Optimal Lyapunov Networking algorithms. The crux of this approach lies in the generation of queue backlog gradients that decrease towards the sink, where these queue backlogs encode certain utility and penalty information. Using information about queue backlogs and link states, nodes can make source rate, packet routing, and forwarding decisions without the notion of end-to-end routes[2].

In this paper, we present the first DTN routing algorithm based on the backpressure approach, a water filling style gradient to deliver data along paths. Our new algorithm combines new ideas with random walk and data packet scheduling approaches.

\section{ROUTING ALGORITHM WITH BACK- PRESSURE APPROACH}

Our new algorithm, which is the first ever backpressure implementation in DTN routing, combines a number of ideas from various approaches: data packet scheduling, random walks, link estimation, and more. From a data packet scheduling perspective, our queue will be based on WFQ. Each new message in node $v$ includes the following parameters: priority and delay in queue. Considering its parameters, node $v$ will insert the new message with a delay value that is equal to its priority into the queue. Each constant time, node $v$ will update all its messages (delay $+=$ priority). When a connection is established, node $v$ will choose to transmit the message in its queue with the greatest delay. In order to choose the neighbor to send the data, each node $v$ computes the new backpressure weight $w_{v, u}$ function for all its neighbors every constant time. Node $v$ sorts the links from the largest value of the backpressure weight, and then normalizes only the links with $w_{v, u}>0$, to a probability between 0 and 1 . Finally, using the probability numbers, node $v$ chooses one of its nodes to forward the message. In case all $w_{v, u}<0$, the node waits until the next update session. In cases where the network allows replication of messages, we 


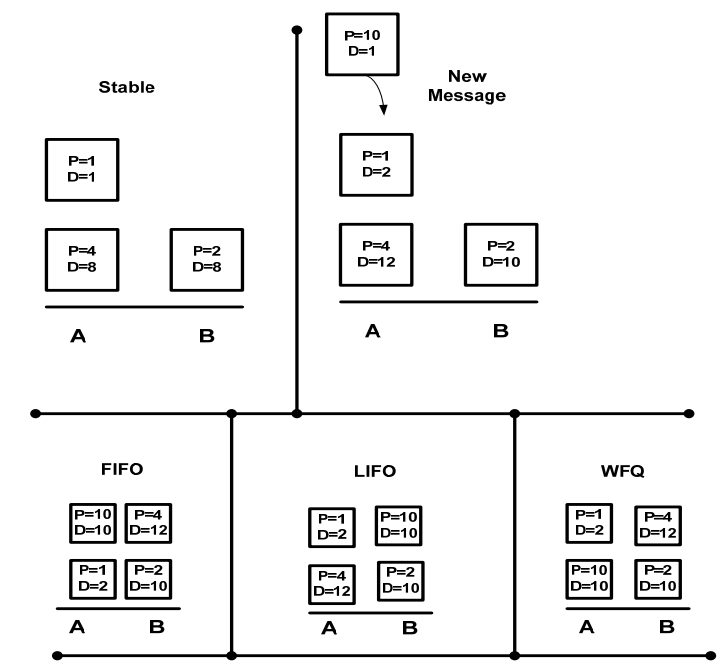

Figure 1: A new message behavior in FIFO, LIFO, and WFQ techniques

can use the new algorithm presented by [3]. We also use a willingness parameter between neighboring nodes. To conclude, after recalculating the weighted queue function for every message in the buffer, we need to calculate the following function based on the ideas above (policy function):

$$
w_{i, j}=\left(\Delta Q_{i, j}-V \cdot \frac{1}{P R R_{i, j}}\right) \cdot \overline{F B E_{i, j}} \cdot S e l F
$$

where $Q_{i, j}=Q_{i}-Q_{j}$ is the queue differential (backpressure); with $Q_{i}$ and $Q_{j}$ representing the backlog of nodes $i$ and $j$, respectively. The $V$ parameter is a constant that trades system queue occupancy for penalty minimization[2]. Packet Reception Rate (PRR) is used for the routing decision with the proposed formula. $F B E$ is based on the Four-bit estimation algorithm. SelF is social selfishness that affects the node behaviors. A node will not forward packets received from those with whom it has no social ties. Therefore, our DTN routing algorithm takes social selfishness into consideration. Finally, after node $v$ calculates the policy function for each of its neighbors, node $v$ normalizes the values in order to choose the neighbor with $w_{i, j}>0$, randomly based on the normalized values. A small example about the difference between WFQ, LIFO, and FIFO behavior can be found in Figure 1. The main parts of the algorithm are: every period of time, each node updates its messages considering their priority ( delay $+=$ priority). Then, a node calculates for any node in its transmission range the policy function $\left(w_{i, j}\right)$ and normalizes the $w_{x, y}$ values. Finally, the node is choose the neighbor to transmit using the random walk algorithm and sends the greatest delay message (Note, an Ack message can be considered a new message).

\section{SIMULATION}

We implemented a testbed based on ONE simulator [1] to evaluate our approach in a variety of test environments. First, we tested an epidemic routing with different queue functions (LIFO, FIFO, WFQ, Random), from the results we can see the best delivery ratio is when the queue function

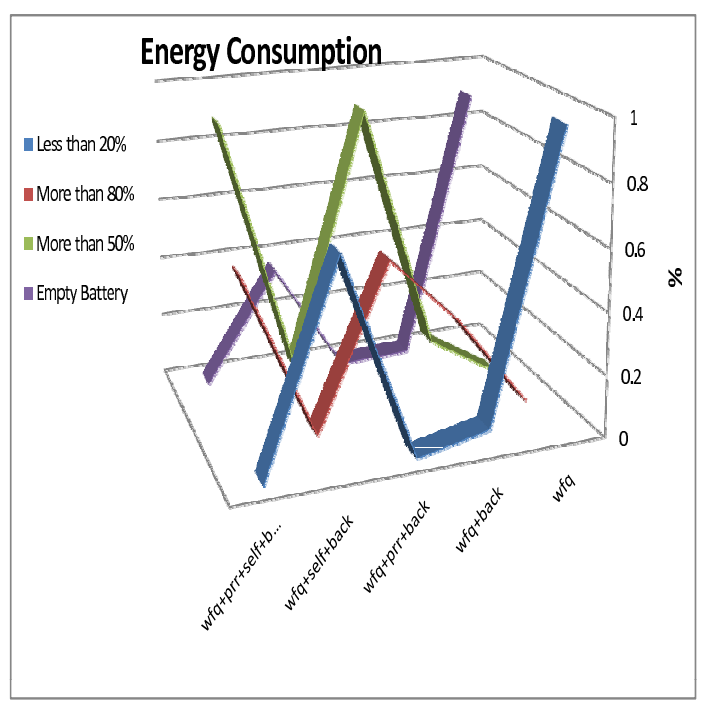

Figure 2: Percentage of Energy Consumption of the nodes

is WFQ. In Figure 2 we used an energy version of epidemic while considering the distance between the nodes. From those results we can see that using only the backpressure approach, backpressure with PRR, backpressure with PRR and queue function gives a small decrease in the delivery ratio $(2 \%)$ but a huge improvement in the energy consumption. In the case we are using only WFQ as our major forwarding algorithm, we get huge improvement in energy consumption while our latency is similar to LIFO results.

\section{CONCLUSION AND FUTURE WORK}

The contributions of this paper include adoption of the backpressure approach into the DTN routing algorithm. Moreover, we showed that combining the backpressure approach with WFQ and PRR approaches improves the efficiency of the energy consumption with a small penalty in the delivery ratio. In future work, we will extensively compare results with existing routing schemes. Moreover, we would like to test the simulation with a more realistic movement model and obtain a greater understanding about the influence of the selfishness, random walk, and replication approaches.

\section{REFERENCES}

[1] A. Keränen, J. Ott, and T. Kärkkäinen. The ONE Simulator for DTN Protocol Evaluation. In Proceedings of the 2nd International Conference on Simulation Tools and Techniques, March 2009.

[2] S. Moeller, A. Sridharan, B. Ksrishnamachari, and O. Gnawali. Routing without routes: The backpressure collection protocol. In Information Processing in Sensor Networks, pages 1-12, 2010.

[3] N. Thompson, S. C. Nelson, M. Bakht, T. Abdelzaher, and R. Kravets. Retiring replicants: Congestion control for intermittently-connected networks. In IEEE infocom, pages 1-9, 2010. 